\title{
О СКОРОСТИ РАСПРОСТРАНЕНИЯ ПРИРОДНОГО ВОЗМУЩЕНИЯ НАПОРА ВДОЛЬ ПОТОКА ПОДЗЕМНЫХ ВОД
}

При анализе пьезометрического режима подземных вод нередко возникает проблема: с жакой скоростью распроспраняется вдоль водоносного горизонта изменение гидравлического напора, обусловленное в некотором сечении пласта внешними природными факторами (колебанием уровня поверхностных водоемов, переменной инфильтрацией или испарением и т. д.). Изучим этот вопрос в одномерной постановке.

Рассмотрим ограниченный в интервале $0 \leqslant x \leqslant L$ фильтрационный поток с напорной поверхностью, который вмещается в однородном по проницаемости пласте, заключенном между водоупорными слоями, причем источник напора между краями пласта отсутствует. Нестационарное распределение гидравлического напора $H(x, t)$ в таком случае описывается дифференциальным уравнением (Бер, Заславски, Ирмей, 1971)

$$
a^{2} \frac{\partial^{2} H(x, t)}{\partial x^{2}}=\frac{\partial H(x, t)}{\partial t}
$$

где $a^{2}-$ коэффициент пьезопроводности.

Примем, что в некоторый начальный момент времени $t=0$ напор в пласте одинаков и равен $C$. В сечении потока $x=0$ напор остается все время постоянным и также равным $C$. В сечении $x=L$ напор изменяется и является некоторой заданной функцией $H_{L}(t)$ времени. Таким образом, начальное условие для потока имеет вид

$$
\left.H(x, t)\right|_{t=0}=\text { const }=C,
$$

а граничные условия

$$
\left.H(x, t)\right|_{x=0}=\text { const }=C,\left.\quad H(x, t)\right|_{x=L}=H_{L}(t) .
$$

Решением уравнения (1) при условиях (2), (3) является формула (Валлнер, 1968)

$$
\begin{gathered}
H(x, t)=C+\left[H_{L}(t)-C\right] \frac{x}{L}+\frac{2 L^{2}}{a^{2} \pi^{3}} \sum_{i=1}^{\infty} i^{-3}(-1)^{i} \sin \frac{i \pi x}{L} \times \\
\times\left\{\frac{\partial}{\partial t}\left[H_{L}(t)-\left.H_{L}(t)\right|_{t=0}\right] \exp \left[-\frac{a i \pi}{L}\right)^{2} t\right]-
\end{gathered}
$$




$$
\left.-\int_{0}^{t} \frac{\partial^{2} H_{L}(\bar{t})}{\partial \bar{t}^{2}} \exp \left[\left(\frac{a i \pi}{L}\right)^{2}(\bar{t}-t)\right] d \bar{t}\right\}
$$

где $i=1,2, \ldots$

В рассматриваемом случае все значения $H_{L}(t) \neq C$ должны, очевидно, обусловливать в интервале $0<x<L$ изменение состояния $H(x, t)=C$. Естественно ожидать, что при этом возмущение напора распространяется вдоль пласта от места его возникновения в сечении $x=L$ с некоторой, в общем переменной, скоростью.

Здесь можно упомянуть об одном осложнении теоретического характера. Судя по выражению (4), всякое изменение граничного условия $H_{L}(t)$ мгновенно отражается в любой точке потока в интервале $0<x<L$, хотя при этом на большом расстоянии от сечения $x=L$ изменение напора и может быть сколь угодно малым. Однако это следствие является лишь математической фикцией, обусловленной неточностью физических предпосылок при выводе фундаментального уравнения (1). Если иметь в виду, что на практике представляют интерес не любые изменения напора в водоносных горизонтах, а только такие, которые могут быть обнаружены имеющимися методами измерений, то на это теоретическое осложнение мы можем не обращать внимания.

Абсолютная погрешность установления уровня воды в колодцах в зависимости от условий измерения составляет обычно $1-3$ cм. Обозначим среднее арифметическое значение ее, принятое равным 2 cм, через $\Delta H$. В нашем случае при изменении состояния $H(x, t)=C$ величину $H(x, t)=C+\Delta H$ целесообразно принять в качестве границы между возмущенной и невозмущенной областями пласта, и назвать здесь фронтом волны возмущения. Скорость передвижения v этого фронта возмущения и будет тогда выражать реальную скорость передачи изменений напора вдоль пласта.

Для установления величины $v$ нами по формуле (4) была произведена серия расчетов, в которых варьировались значения коэффициента пьезопроводности и скорости изменения напора на границе пласта. Напор $H_{L}(t)$ задавался при этом в виде

$$
H_{L}(t)=A(1-\cos 2 \pi t / T)+C,
$$

где $A-$ амплитуда и $T$ - период его изменения.

В таком случае средняя скорость $\bar{v}$ возрастания напора на границе пласта выразится зависимостью $\bar{v}=2 A / T$. Откуда, если использовать значения $T=1 ; 10$ и 100 сут и $A=1$, получим соответственно $\bar{v}=2,0 ; 0,2$ и $0,02 \mathrm{~s} / \mathrm{cy}$.

В природных условиях величина $\bar{v}=0,02 \mu / c y т$ характеризует весьма медленное изменение гидравлического напора, величина $\bar{v}=0,2 \mathrm{M} /$ cyт $_{-}$ умеренное, а $\bar{v}=2,0 \mathrm{\mu} / \mathrm{cyt}$ - весьма интенсивное изменение напора.

Значение коэффициента пьезопроводности $a^{2}$ бралось при расчетах в его реальных пределах - от $10^{5}$ до $10^{7} \mathrm{M}^{2} / c y T$. Длина потока $L$ была принята равной $10 \kappa м$.

Произведенные вычисления (см. таблицу и рисунок) позволили придти к следующим общим выводам.

Скорость передвижения фронта возмущения тем больше, чем быстрее происходит приращение напора в граничном сечении потока и чем выше пьезопроводность водоносного горизонта.

При весьма медленном изменении напора на границе потока возму. щение передается вдоль водоносного горизонта со скоростью $0,1-0,7$ 


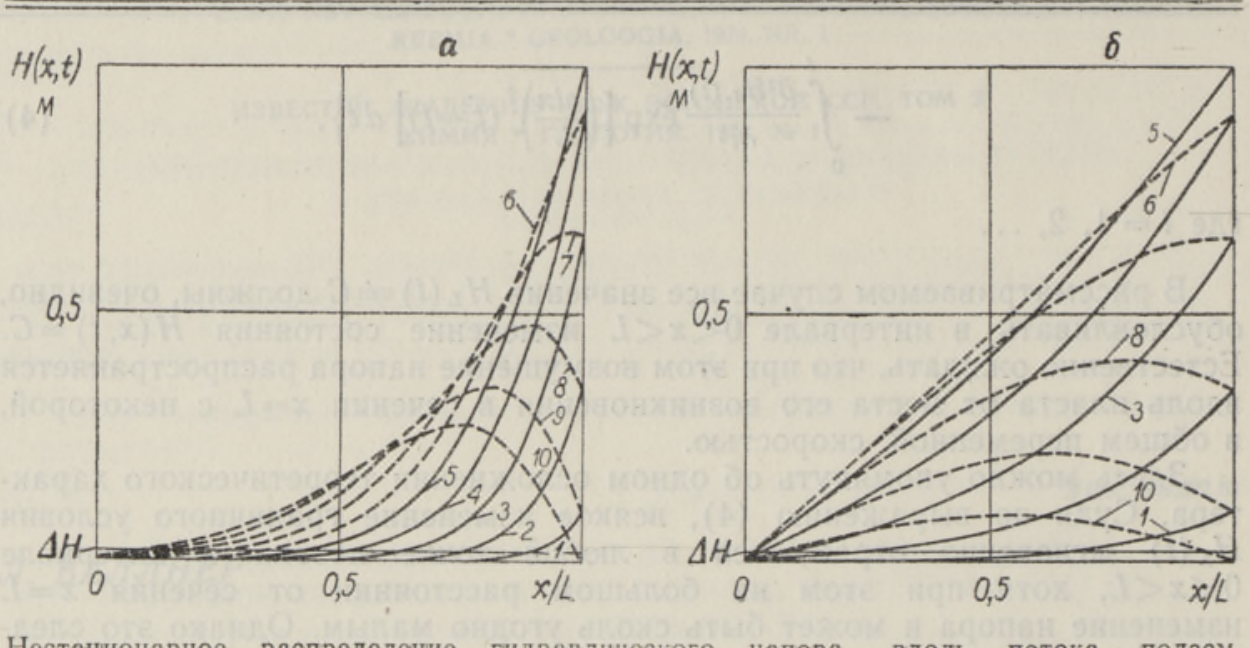

Нестационарное распределение гидравлического напора вдоль потока подземных вод при $a^{2}=10^{6} \mathrm{M}^{2} / c y T ; a-T=10$ сут, б-T=100 cyт.

$l-t=0,1 T ; 2-t=0,2 T ; 3-t=0,3 T ; 4-t=0,4 T ; 5-t=0,5 T ; 6-t=0,6 T$; $7-t=0,7 T ; 8-t=0,8 T ; 9-t=0,9 T ; 10-t=T$.

Скорость передвижения фронта возмущения $v(\kappa м / \mathrm{cyT})$

в зависимости от средней скорости возмущения на границе потока $(\vec{v})$ и коэффициента пьезопроводности горизонта $\left(a^{2}\right)$

\begin{tabular}{c|r|rr}
\hline \multirow{2}{*}{$a^{2}, \mu^{2} / c y T$} & \multicolumn{3}{|c}{$\bar{v}$, M/cyT } \\
\cline { 2 - 4 } & 0,02 & 0,2 & 2,0 \\
\hline \multirow{2}{*}{$0^{5}$} & 0,1 & 0,4 & 1,2 \\
$10^{6}$ & 0,3 & 1,2 & 3,6 \\
$10^{7}$ & 0,7 & 3,0 & 11,0
\end{tabular}

$\kappa м / с у т$ в зависимости от пьезопроводности. Умеренные по своей интенсивности изменения граничного условия обусловливают уже более быстрое передвижение фронта возмущения, достигающее $3,0 \kappa м / c y T$. Весьма интенсивные граничные возмущения напора распространяются c наибольшей скоростью, которая может превышать $11 \kappa м / c y т$.

Общая тенденция такова, что десятикратное увеличение либо интенсивности граничного возмущения потока, либо пьезопроводности водоносного горизонта ускоряет передвижение фронта возмущения примерно в три-четыре раза.

Согласно формуле (5) напор на границе потока приобретает максимальное значение к моменту времени $t=T / 2$, а потом монотонно убывает до своей первоначальной величины - таков в первом приближении характер природных колебаний гидравлического напора. Однако может случиться, что фронт возмущения при возрастающей стадии напора $H_{L}(t)$ еще не успевает достичь сечения пласта $x=0$ (рисунок). Тогда при $T / 2<t<T$, т. е. на убывающей стадии напора $H_{L}(t)$, фронт возмущения будет продолжать перемещаться к сечению пласта $x=L$, хотя в среднем и не так быстро, как в промежутке времени $0<t<T / 2$.

$\mathrm{K}$ моменту $t=T$ по условию (5) в сечении пласта $x=L$ восстанав. ливается величина напора $C$. Однако несмотря на это, возмущенная зона в водовмещающем пласте сохраняется. Распределение напора в ней определяется выражением (4), причем кривая функции $\left.H(x, t)\right|_{t=T}$ имеет выпуклую форму. В течение времени $t>T$, если только граничные условия потока не изменятся, распределение напора вдоль него будет постепенно выравниваться. Этот процесс описывается формулой

$$
\left.H(x, t)\right|_{t>T}=\sum_{i=1}^{\infty}\left[\frac{2}{L} \sin \frac{i \pi x}{L}\right] \exp \left[-\left(\frac{a i \pi}{L}\right)^{2} t\right] \int_{0}^{L} H(x, T) \sin \frac{i \pi x}{L} d x .
$$


Выравнивание напора, происходящее по экспоненциальному закону, теоретически должно длиться бесконечно долго. На самом же деле, особенно при высоких значениях пьезопроводности пласта, величина превышающего значение $C$ остаточного напора может весьма быстро уменьшаться до своего практически ощутимого предела $\Delta H$.

\section{Л ИТ Р РАТ У Р А}

Бер Я., З асл авски Д., Ирмей С. 1971. Основы фильтрации воды. М.

В а ллн е р Л. 1968. Нестационарный режим фильтрации одномерного потока подземных вод. Изв. АН ЭССР, Хим. Геол., 17, № 1.
Институт геологии
Академии наук Эстонской ССР
Поступила в редакцию $22 /$ VI 1973

\section{VALLNER}

\section{ROHU LOODUSLIKU ERGASTUSE LEVIKU KIIRUSEST POHJAVEE vooLUS}

Mittestatsionaarne ühemõōtmeline hüdraulilise rōhu jaotus põhjavee voolus on tingimustel (2), (3) ja (5) arvutatav valemi (4) abil. Rōhu muutumise frondi liikumise kiirus $v$ sõltub ergastuse intensiivsusest $\bar{v}$ ja veehorisondi piesojuhtivusest $a^{2}$ (tab.).

\section{VALLNER}

\section{ON THE MOVEMENT VELOCITY OF THE NATURAL CHANGE OF THE HYDRAULIC PRESSURE ALONG THE GROUND WATER FLOW}

The mon-steady one-dimensional distribution of the change of hydraulic pressure is calculated by formula (1) under conditions (2), (3) and (5). The velocity $v$ of movement of the pressure $H(x, t)=C+\Delta H(\Delta H=0,02 \mathrm{~m})$ along the flow (Fig.) depends on the mean velocity $\bar{v}$ of the change of the boundary condition (5) and $a^{2}=T / S$, where $T$ is the coefficient of the transmissivity and $S$ - the coefficient of the storage of aquifer (Table). 